\title{
The future of refractive surgery: presbyopia treatment, can we dispense with our glasses?
}

\author{
Parwez Hossain $\mathbb{1}^{1,2} \cdot$ Ramez Barbara ${ }^{1,3}$
}

Received: 30 July 2020 / Revised: 7 August 2020 / Accepted: 25 August 2020 / Published online: 7 September 2020

(c) The Royal College of Ophthalmologists 2020

Refractive error remains the leading cause for visual impairment. With an aging world population, presbyopia is the leading refractive deficit. In many parts of the developed world, the average person can spend half of their lives in presbyopia [1]. With increasing digital demands, there is an increasing dependence on accommodation and presbyopic symptoms can appear as early as the 30s age group [2]. With refractive error affecting most of the world's population at some point in their lives, it is not surprising that surgical approaches to correct refractive error have gained interest. Over the past 30 years, we saw an explosion in the variety of surgical approaches with their own relative nuances in technique, risks and benefits [2].

In this edition, Ang et al. [3], overview the advancements in refractive surgery over the past three decades and provide an insight, beyond 2020 . The authors cover the rich variety of procedures, showing how surgery has evolved and how it can correct vision beyond just the sphere and cylindrical corrections. Refractive laser surgery can reshape the cornea to reduce high-order aberrations, improving glare and night vision [4-6].

Ang et al. [3] also show the individual advancements in techniques such as in PRK, outlining corneal surface ablation approaches or as in LASIK with transition from microkeratome to femto-second laser assisted corneal flaps formation. In addition, they overview the merits of most recent advances of minimally invasive laser eye treatmentSMILE. The authors highlight previous evaluative studies

Parwez Hossain

parwez@soton.ac.uk

1 Southampton Eye Unit, University Hospitals Southampton, Southampton, UK

2 Clinical Experimental Sciences, Faculty of Medicine, University of Southampton, Southampton, UK

3 Birmingham Midlands Eye Centre, Birmingham, UK that show patients are satisfied with laser surgery outcomes, however, most of this is with LASIK to correct myopia [7].

Refractive surgery has yet to be so convincing, in the realm of presbyopia therapy. With over 2.1 billion presbyopes worldwide, this is one of the world's important but unconquered health issues. The demand is great. As mentioned earlier, with a rising proportion of presbyopes in the population, one can expect to live 40 years or more in this refractive state! [1].

Currently, traditional approaches to correct presbyopia are to use single-vision near, bifocal, and progressive spectacle lenses. Multifocal contact lenses also allow presbyopic correction. It is interesting to note that spectacle lens technologies are also advancing. Spectacle lens technologies have the advantage and flexibility of correcting progressive presbyopia by providing regular updates of the refractive correction [8]. Over the past decade, we have seen widespread use and take-up of spectacle technologies developed by lens manufacturers, such as use of 'free form digital' progressive lenses. These lenses allow tailored individualised presbyopic correction. Offering 'pseudodynamic accommodation' with eye seeing through wider optical zones. This is often cited by manufacturers as ideal for our 'modern presbyope' who needs to have a single pair of glasses that gives clear vision at a variety of viewing distances such as moving from computer screens to mobile phones and vice versa. Due to commercial sensitivities, it is difficult to find peer reviewed studies that substantiate the manufacturers' claims but these lenses appear popular [2].

The future challenge for surgical refractive techniques for presbyopia is whether there is a technique that genuinely helps to emulate the dynamic features of the accommodative process? [2]. Currently, we have several surgical strategies. Most of them are compromises, such as producing monovision with refractive lens exchange with appropriate intraocular lens implants or laser refractive surgery to achieve a difference in ocular dioptric powers between the eyes. Alternatives to monovision include simultaneous images for distance and near with special 
intraocular lenses or use of corneal inlays. Other approaches to correct presbyopic symptoms include, using pinhole depth of focus expansion by using appropriate intraocular lenses or corneal inlays. Accommodative lens implants, multifocal implants and extended depth of focus lens implants can all be used but each have their limitations [2]. There are other approaches that offer 'true' restoration of accommodation but are not proven such as laser crystalline lens softening or use of 'accommodating' intraocular lenses.

Ang et al. review [3], covers a variety of surgical approaches to deal with presbyopia with a large focus on corneal inlay technologies. Keratophakia was first kind of corneal inlay technology, described as a far as 1964 for the treatment for both hyperopia and presbyopia, it forms the basis for inlay surgery [9]. Since then, there has been significant modification and advancement of inlay technology over the years, overcoming previous issues with maintaining transparency, corneal nutrition and oxygen diffusion. Fewer develop corneal melting and extrude.

Generally, there are three types of corneal inlays. First, inlays that create a multifocal cornea by reshaping the anterior curvature, second, a multifocal with a bifocal optic and finally inlays that improve depth of focus by creating a small aperture based on pinhole [10]. There are various models available (Flexivue Microlens, Icolens, Raindrop and Kamra Vision) and although corneal inlays have not achieved the same impact and popularity as laser refractive surgery for the correction of myopia. Small aperture inlays (Kamra) seem to provide very good near and intermediate vision without compromising distance vision, contrast sensitivity and stereopsis especially in emmetropes and hyperopes [9-12]. Despite potential advantages corneal inlays still possess, a small but considerate rate of dissatisfaction for a refractive procedure.

Further advantages of corneal inlays are that they have an additive effect with no tissue removal and are reversible. They also preserve the recipient ability to have other additional surgical refractive procedures. They have enhanced safety profile compared to intraocular surgery. With the more modern inlay designs, there are fewer stromal cellular changes or inflammation and they have no issues with visual field scotomas or visualisation of the retina $[12,13]$. In terms of surgery, surgical centration appears to be crucial, to avoid reduced image quality [9]. Dissatisfaction is mainly due to glares and halos, dry eyes and night vision problems, which tend to be mostly mild to moderate.

Another further approach using inlay technology is for scleral expansion surgery. Scleral expansion has been suggested as a method to restore dynamic accommodation by increasing the distance between the lens equator and the ciliary body. Whether this is indeed the case remains controversial. Initial surgical series show some improvement in the amplitude of accommodation. Currently, the VisAbility Micro-Insert scleral implant (Refocus Group, Dallas, TX, USA) has a CE mark and is currently undergoing clinical trials [14]. However, there are risks from anterior segment ischaemia, implant extrusion to the subconjunctival space and infective complications.

SMILE surgical procedures have produced the opportunity for new 'biological inlays'. The extracted corneal lenticels following a SMILE procedure could be used as 'allogenic transplant' intracorneal inlays to correct other patients' with refractive errors. Theoretically, the higher biocompatibility of using human tissue potentially reduces the risks and complications associated with synthetic inlays. However, there remain Issues with post-operative remodelling of the transplanted lenticel and the host stroma with significant under-correction of refractive error. Furthermore, there are risks of allograft rejection and potential viral transmission from graft to host. Some have suggested these could be overcome using decellularized lenticular corneal material, procured/processed by eye banks [3]. There is need to develop an integrated clinical governance pathways that apply same stringent control measures used in eye/tissue banks.

Returning to the discussion, do these treatments solve presbyopia? Intracorneal inlays for the treatment of presbyopia show some level of correction by increasing the patient's depth of field. We also have other surgical options such as monovision with laser or following cataract surgery. Recent attempts at reshaping the cornea to create a multifocal cornea and/or to achieve laser blended vision to achieve extended depth of focus have not gained popularity or the desired level of spectacle independence [15, 16]. These may suit some who seek spectacle independence. However, none are close to 'dynamic' physiological correction for presbyopia and the outcomes show that they are some way off improving near vision. Would there be a treatment that can restore natural dynamic features of accommodation and retain binocular near vision? Would there be a surgical approach that can overcome the progressive age-related reductions in the amplitude of accommodation and the need to regularly increase the near refractive correction?

In summary, we have a wealth of approaches that seek to address presbyopia. When considering surgical interventions, we are dealing with physiologically healthy eye that has the ability of achieving clear vision with non-surgical means. These patients are looking at improving their quality of vision and have high expectations. We must not forget that non-surgical optical technologies are also advancing in terms of spectacle lens designs and contact lenses, these offer flexibility of regular updates for refractive changes etc. So as ever there is no easy solution to get rid of presbyopia, 
let's look forward to see what advancements hold up in this century?

\section{Compliance with ethical standards}

Conflict of interest Parwez Hossain is a deputy leader for Ocular Tissue Advisory Group, NHS Blood \& Transplant, UK; Royal College of Ophthalmologists, Regional Representative, Advisory work for Parapharm, Santen, Novartis, Thea Pharmaceuticals. He has received non-financial benefits from Zeimer Ophthalmic Lasers.

Publisher's note Springer Nature remains neutral with regard to jurisdictional claims in published maps and institutional affiliations.

\section{References}

1. Holden BA, Fricke TR, Ho SM, Wong R, Schlenther G, Cronje S, et al. Global vision impairment due to uncorrected presbyopia. Arch Ophthalmol. 2008;126:1731-9.

2. Wolffsohn JS, Davies LN. Presbyopia: effectiveness of correction strategies. Prog Retin Eye Res. 2019;68:124-43.

3. Ang et al. Refractive Surgery Beyond 2020. Eye (Lond). 2020. https://doi.org/10.1038/s41433-020-1096-5. Online ahead of print.

4. Seiler T, Genth U, Holschbach A, Derse M. Aspheric photorefractive keratectomy with excimer laser. Refract Corneal Surg. 1993;9:166-72.

5. O'Brart DP, Corbett MC, Lohmann CP, Kerr Muir MG, Marshall $\mathrm{J}$. The effects of ablation diameter on the outcome of excimer laser photorefractive keratectomy. A prospective, randomized, doubleblind study. Arch Ophthalmol. 1995;113:438-43.

6. Kalski RS, Sutton G, Bin Y, Lawless MA, Rogers C. Comparison of 5-mm and 6-mm ablation zones in photorefractive keratectomy for myopia. J Refract Surg. 1996;12:61-7.
7. Sugar A, Hood CT, Mian SI. Patient-reported outcomes following LASIK: quality of life in the PROWL studies. JAMA 2017;317:204-5. https://doi.org/10.1001/jama.2016.19323.

8. Charman WN. Developments in the correction of presbyopia I: spectacle and contact lenses. Ophthalmic Physiol Opt 2014;34:8-29.

9. Lindstrom RL, Macrae SM, Pepose JS, Hoopes PC Sr. Corneal Inlays for presbyopia correction. Curr Opin Ophthalmol. 2013 Jul;24:281-7.

10. Tomita M, Kanamori T, Waring GO 4th, et al. Simultaneous corneal inlay implantation and laser in situ keratomileusis for presbyopia in patients with hyperopia, myopia, or emmetropia: six-month results. J Cataract Refract Surg. 2012;38:495-506.

11. Dexl AK, Seyeddain O, Riha W, et al. One-year visual outcomes and patient satisfaction after surgical correction of presbyopia with an intracorneal inlay of a new design. J Cataract Refract Surg. 2012;38:262-9.

12. Seyeddain O, Hohensinn M, Riha W, et al. Small-aperture corneal inlay for the correction of presbyopia: 3-year follow-up. J Cataract Refract Surg. 2012;38:35-45.

13. Brooker ET, Waring GO IV, Vilupuru AS, Sanchez Leon F. Effect of small aperture intra-corneal inlay on visual fields. Invest Ophthalmol Vis Sci. 2012;53:1391.

14. U.S. National Institutes of Health Clinical Trials. A clinical trial of the VisAbility Micro Insert System for presbyopic patients. https://clinicaltrials.gov/ct2/show/NCT02374671.

15. Holland D. PresbyLASIK treatment for simultaneous correction of presbyopia and ametropia: development to PresbyMAX hybrid at the Augenklinik Bellevue. ESCRS; 2014. https://www.escrs.org/ london2014/programme/free-papers.asp? $\mathrm{id}=519 \&$ day $=0$.

16. Rocha KM, Vabre L, Chateau N, Krueger RR. Expanding depth of focus by modifying higher-order aberrations induced by an adaptive optics visual simulator. J Cataract Refract Surg. 2009;35:1885-92. 